\title{
Fast Intra Prediction Mode Decision for High Efficiency Video Coding
}

\author{
Haijun Lei \\ College of Computer Science \& Software Engineering \\ Shenzhen University \\ Shenzhen City, China \\ e-mail:1hj@szu.edu.cn
}

\author{
Zhongwang Yang \\ College of Computer Science \& Software Engineering \\ Shenzhen University \\ Shenzhen City, China \\ e-mail:tzwydragon@163.com
}

\begin{abstract}
High Efficiency Video Coding (HEVC) is the latest video compression standard for high resolution video content. It is said that HEVC promises the significant compression performance improvement compared to the H.264/AVC. However,it comes with the tremendous encoding complexity increase. It is necessary to decrease the computation complexity of coder. In this paper, after analysis of the coding unit and prediction unit algorithm, a fast mode decision scheme for intra prediction is proposed. This algorithm calculates firstly the texture complexity of coding unit, and set the rational threshold to select the size of coding unit fast; then a modified search process is used to decrease the number for candidate modes of prediction,finally early termination for rate-distortion optimized quantization(RDOQ) is used for Intra prediction. The simulation results showed that,compared with the recent HEVC conference software, the proposed algorithm can reduce about $\mathbf{4 0 . 9 \%}$ encoding time while suffers from a negligible on bit-rates performance and a negligible average PSNR loss.
\end{abstract}

Keywords-High Efficiency Video Coding; HEVC; coding unit; prediction unit; context complexity; RDOQ

\section{INTRODUCTION}

The use of high-definition(HD) broadcast and television services above HD-level has become widespread, aided by rapid progress in multimedia processing technology. Recently, under the efforts of Joint Collaborative Team on Video Coding (JCT-VC),high efficiency video coding (HEVC) was established[1], in order to set up a new, more efficiency and state-of-the-art video compression standard.

HEVC is still under the traditional block-based hybrid coding frame, but it introduces a large number of innovations, including the recursive tree structure, multi-angle intra prediction, large block transforms, advanced motion estimation, adaptable loop filtering operations and etc[2][3][4]. The macro-block concept in H.264/AVC has been replaced by the Coding Unit (CU), and then each CU is split into sub-CUs, Each leaf sub-CU can be further split into many entities are called prediction units (PU) and transform units(TU).In the intra prediction process, both $\mathrm{CU}$ and $\mathrm{PU}$ are squared size with one $2 \mathrm{Nx} 2 \mathrm{~N}$ unit or four $\mathrm{NxN}$ sub-unit, where $\mathrm{N}$ is one of the array[4,8,16,32].Besides, $\mathrm{TU}$ is also recursively imple- mented for residual coding and incurs more complexity requirement. Figurel gives the based recursive structure illustration for $\mathrm{CU}, \mathrm{PU}$ and TU. For detail description, please refer the[1].

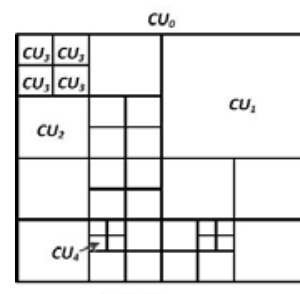

(a) CU Structure

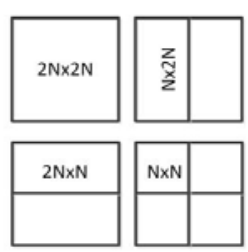

(b) PU Structure

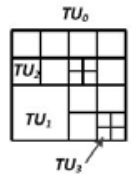

(c) Tu Structure
Figure1. CU, PU and TU splitting structure in HEVC

Intra coding in HEVC can be considered as an extension of H.264/AVC, as both approaches are based on spatial sample prediction followed by transform coding. However, In HEVC, there are 35 directional intra prediction candidates, where mode 0 is Planar mode, mode 1 is DC mode and 2 to 34 are angular prediction modes with different directions[5], as shown in Figure 2. It requires so much computing resource to recursively conduct full Rate-Distortion Optimized Quantization (RDOQ) for all modes at different PU levels. In HEVC reference software, a three-step mode decision process is adopted [6]. Firstly, rough mode decision is used for the mode candidates; and then checking the most possible mode(MPM); Furthermore, recursive transforms are applied to the optimal mode from the first two stages for the residual coding to derive the final best coding mode. Though the method can get a nice video quality, the calculate complexity still quite high for application.

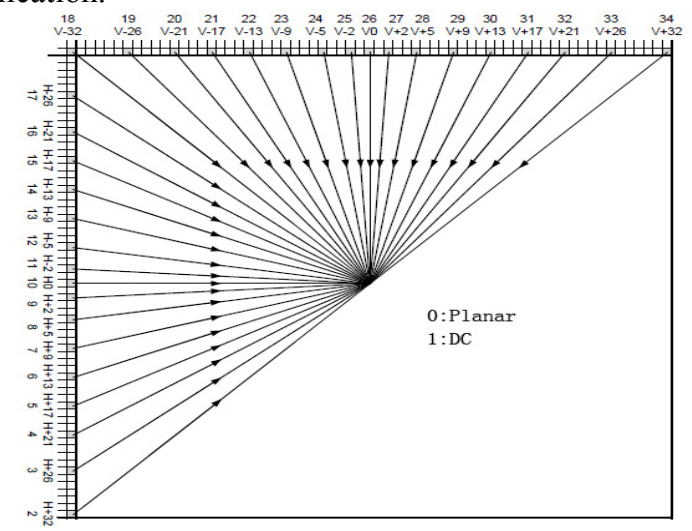

Figure2. Intra prediction modes

The rest of the paper is organized as follows. Section 2 
review related works for intra predictions. Section 3 introduces the proposed fast intra prediction algorithm. Section 4 presents the simulation result, and our conclusions are given in Section 5.

\section{RELATED WORKS}

Fast intra mode decision has been extensively studied for H.264/AVC, it is also the important research point for decreasing the complexity in HEVC. However, these algorithms for H.264/VC can not be directly applied to the HEVC due to so many different coding structure and prediction modes. Recently, some fast intra prediction algorithms are proposed for HEVC.

In reference [7], Tan et al. proposed a fast RQT algorithm for both intra and inter mode coding in order to reduce the calculating complexity. In [7], for all intra case, encoding time can be saved $13 \%$, however, BD-Rate justly increases $0.1 \%$. For random access and low delay constraint, it reduces up to $9 \%$ encoding time with $0.3 \%$ BD-Rate performance degradation.

J. Lainema et al. proposed an intra prediction method which is designed to provide high compression efficiency and which can be implemented effectively in resource constrained environments making it applicable to wide range of use cases[8]. That algorithm outperforms the prior H.264/AVC on average $4.8 \%$ and exceeds $10 \%$ in the dominant directional structures.

Liang Zhao et al. proposed a fast mode decision by reducing the candidates in RDO process [9]. In that proposal, most probable mode (MPM) is included for RDO in order to reduce the number of the candidates. Besides, the experimental results show the proposed scheme provides $14 \%$ and $20 \%$ time saving in intra high efficiency and low complexity cases compared to the default encoding scheme in HM0.9, and adopted into the HEVC test model.

Reference [10] presents a fast prediction method based on edge information of the current PU. It chooses a reduced set of directions so as to decrease the cost of very high computational complexity. Final experimental results show that algorithm can provide a decrease of up to $32.08 \%$ with negligible PSNR reduction.

Reference[11] exploits the correlation between the prediction directions of neighboring prediction units and that of the encoded prediction unit, and then creating a merger priority list of prediction directions for current PU. The method can improve the efficiency of intra prediction, and minimize the complexity of encoder and decoder.

In reference [12], Guifeng Tian et al. proposed a PU size decision algorithm to speed up the intra coding. In the method, two-stage is applied. In the pre-stage, filtering the unnecessary PU by analyzing the texture complexity of LCU and its four sub-block; secondly, skipping the small PU candidates by referring the neighboring PU. The simulate results present that proposed method can speed up by averagely 44.91, and PSNR degradation less than $0.04 \mathrm{~dB}$.

In reference[13], Hao Zhang and Zhan Ma proposed a fast intra prediction through reduce the count of candidate modes. Firstly, list the initial modes for searching better candidate mode; Secondly, searching the best modes based the search steps; Thirdly, early RDOQ termination. The result of proposed arithmetic achieves $38 \%$ encoding time reduction with less than 3\% BD-Rate increase.

There are many other fast intra prediction algorithms for HEVC, however, aforementioned algorithms and others are mainly focusing on PU size selected with skipping the unnecessary further split or merger process; they don't combine the CU fast algorithms and PU related algorithms for further increasing the coding efficiency.

\section{PROPOSED ALGORITHM}

The proposed method fully considers the CU and PU size or modes decision algorithms. In CU partition, we analysis the CU texture characteristic, in order to decide whether split the current CU. In PU partition, we exploit down-sampling prediction, similar three-step search [14] and early termination for intra prediction so as to reduce the candidate modes and speed up intra prediction. The following is detail of the all process.

\section{A. CU early termination}

It is already proved that when the $\mathrm{CU}$ texture is flat, or the pixels in CU are similar, the CU may not divide sub-units; when the CU texture is complex, the CU generally splits into smaller sub-unit and then finds the best size [12].

In our proposed algorithm, down-sampling method is exploited to decrease the computational complexity and get rid of nose. In Figure 3, the current $\mathrm{CU}$ apply the 2:1 down-sampling filter by a simple average operator $(2: 1$ in direction of horizontal, and 2:1 in direction of vertical), other size CU have the similar operation.
Size: $64 \times 64$

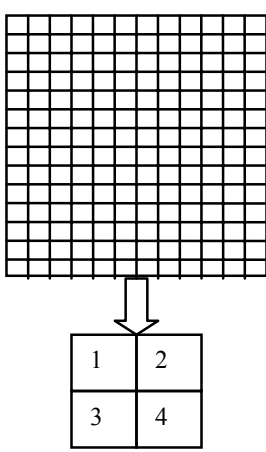

Figure3. Illustration of simple averaging based down-sampling on
After down-sampling, the complexity of the original LCU can be executed as its variance defined as

$$
E_{\text {com }}=\sum_{i=0}^{N-1} \sum_{j=0}^{N-1}\left[p(i, j)-\frac{1}{N} \frac{1}{N}\left(\sum_{i=0}^{N-1} \sum_{j=0}^{N-1} p(i, j)\right)\right]
$$

where $E_{\text {com }}$ represents the texture complexity, $N$ is the size of the current $\mathrm{CU}, p(i, j)$ is the pixel and $(i, j)$ is the coordinate in $\mathrm{CU}$.

Based on the texture calculation, we set the two threshold for tradeoff of coding quality and complexity reduction, they 
are Threl and Thre2.When the complexity is greater than Threl, the CU is split; when the complexity is less thanThre 2, the CU is optimal; When the complexity is between Threl and Thre2, referring the HEVC reference software.

\section{B. Fast PU modes decision}

In HEVC reference software, there are quite computation complexity for selecting a optimal mode from 35 modes. We proposed a fast intra mode decision algorithm to reduce the complexity. The algorithm is formed by two stage; firstly, calculating Sum of Absolute Differences(SAD) by the down-sampling method, and then applying the similar three-step search algorithm to remove unnecessary modes; secondly, RDO early termination. The detail operation of the algorithm is following:

1)Initially, creating candidates list $\mathrm{S} 1=$ $\{0,1,2,6,10,14,18,22,26,30,34\}$ from 35 prediction modes, and then check $\mathrm{S} 1$ so as to select 5 optimal modes by SAD, Suppose the 5 mode are $\mathrm{S} 2=\{0,2,10,18,34\}$.

2)Based on the three-step algorithm, we firstly extend the list S2, the 2-distance neighbors of S2 are $\mathrm{S} 3=\{4,12,20,32\}$ (the mode 0 and 1 are Considered separately), checking theS1,S2 and S3 in order to get the optimal modes, suppose the optimal modes are S4 $=\{6,14,22\}$. Additionally, suppose the modes of upper and left PUs are S5 $=\{1,4\}$, checking $\mathrm{S} 1, \mathrm{~S} 2, \mathrm{~S} 3, \mathrm{~S} 4, \mathrm{~S} 5$ for the optimal modes, suppose the optimal two modes are $\mathrm{S} 6=\{4,6\}$.

3)The 1-distance neighbors of $\mathrm{S} 6$ are $\mathrm{S} 7=\{3,5,7\}$, choosing the best $\mathrm{K}$ modes as the candidates for full RDOQ by checking the list from $\mathrm{S} 1$ to $\mathrm{S} 7$. Suppose $\mathrm{K}$ is 3 and modes are $\{3,5,6\}$, besides, the minimal cost of modes is $\mathrm{Cmin}(\mathrm{Cmin}$ is the cost of mode min); if the cost of mode $\mathrm{m}$ is larger than weighted $\mathrm{Cmin}$, as $\mathrm{C}_{\mathrm{m}}>\alpha \mathrm{C}_{\min }$, and then mode $\mathrm{m}$ does not perform the full RDOQ. If $\mathrm{C}_{\mathrm{m}}<\alpha \mathrm{C}_{\text {min }}$, if the $\mid$ min-m $\mid>3$, skip the mode $\mathrm{m}$; if the $|\min -m|<3$, applying the HEVC reference software algorithm. Other modes are processed similarly.

\section{Integrated fast algorithm}

Our paper considers the $\mathrm{CU}$ and PU, and then sets the threshold, reduces the intra prediction candidates and early termination method etc. Figure 4 is the general process for current CU.

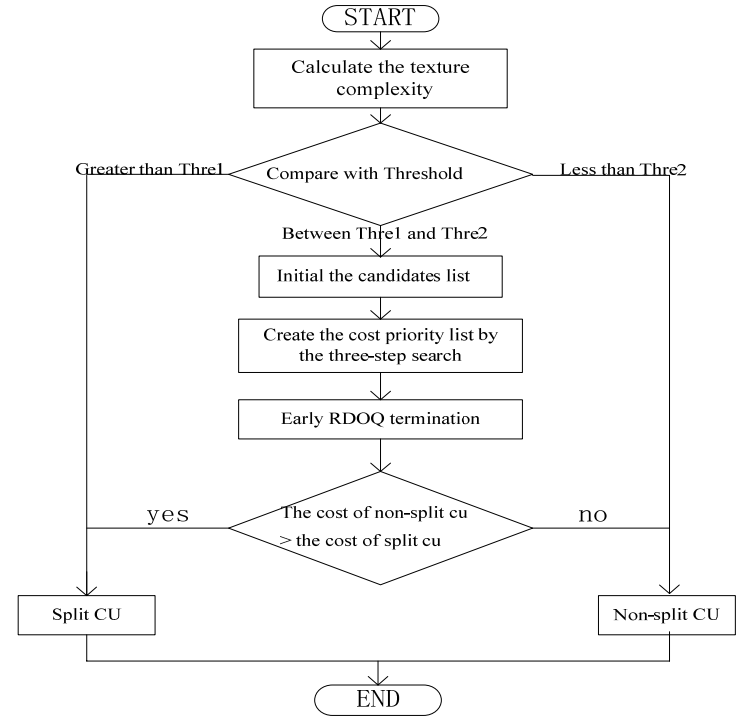

Figure 4. Proposed method for fast intra prediction

\section{Simulation Results}

The proposed algorithm has been implemented into the HEVC reference software HM8.0 [15]. The HEVC test sequences are shown in reference [16] and the common test condition is the constraints of intra-only. In order to compare the performance of proposed algorithm against HM8.0, simulations are carried out using the PC with Intel Pentium E5800@3.2GHz, 2GB memory, and windows 7Professional SP1 32-bit O/S, Quantization parameters are 22,27,32,37. The performances are evaluated based on BD-rate, BD-PSNR and $\Delta \mathrm{t}$. The BD-rate and BD-PSNR are calculated by the tools in [17]; $\Delta \mathrm{t}$ is the coding time reduction and measured by the equation(2).

$$
\Delta t=\frac{\text { Time }_{\text {proposed }}-\text { Time }_{H M}}{\text { Time }_{H M}} \times 100 \%
$$

Where Time proposed $_{\text {and Time }}$ HM represent the time of proposed method and the traditional method time respectively.

Table 1 shows the simulation results by the proposed algorithm. Table 1 reveals the average cost time reduction, the negligible BD-rate increases and the BD-PSNR loss. In view of its complexity, the proposed method saves, on average, $40.9 \%$ cost time, and the biggest complexity saves is $44.1 \%$. The average increases of BD-rate (Luma-Y) are $1.6 \%$ and the average losses of BD-PSNR is $0.05 \mathrm{~dB}$. It is also suggested more time tend to be saved in the low bit rate cases. That is because that under the low bit rate condition larger CUs are efficiency and intra prediction modes are similar.

As the table results, the encoder is more likely adopt larger CUs, and early termination and skipping the unnecessary candidates can get a big time reduction and maintaining the RD performance. 
TABle I. Simulation RESUltS By COMPARING WiTH HM8.0

\begin{tabular}{|c|c|c|c|c|}
\hline class & sequences & $\begin{array}{c}\text { BD-rate } \\
(\%)\end{array}$ & $\begin{array}{c}\text { BD-PSNR } \\
(\mathrm{dB})\end{array}$ & $\overline{\Delta t}(\%)$ \\
\hline \multirow[t]{4}{*}{ A } & NebutaFestival & 0.5 & -0.08 & -41.9 \\
\hline & PeopleOnStreet & 1.8 & -0.02 & -41.2 \\
\hline & SteamLocomotiveTrain & 1.0 & -0.03 & -40.8 \\
\hline & Traffic & 1.3 & -0.04 & -39.5 \\
\hline \multirow[t]{5}{*}{ B } & BasketballDrive & 1.8 & -0.01 & -40.3 \\
\hline & BQTerrace & -0.08 & -0.05 & -43.4 \\
\hline & Cactus & -0.02 & -0.01 & -39.5 \\
\hline & Kimono1 & -0.03 & -0.03 & -41.6 \\
\hline & ParkScene & -0.04 & -0.03 & -40.2 \\
\hline \multirow[t]{4}{*}{$\mathrm{C}$} & BasketballDrill & -0.01 & -0.06 & -44.1 \\
\hline & BQMall & -0.05 & -0.08 & -42.8 \\
\hline & PartyScene & -0.01 & -0.06 & -41.8 \\
\hline & RaceHorses & -0.03 & -0.04 & -43.67 \\
\hline \multirow[t]{4}{*}{$\mathrm{D}$} & BasketballPass & -0.03 & -0.1 & -40.0 \\
\hline & BlowingBubbles & -0.06 & -0.06 & -39.0 \\
\hline & BQSquare & -0.08 & -0.07 & -49.3 \\
\hline & RaceHorses & -0.06 & -0.08 & -39.3 \\
\hline \multirow[t]{3}{*}{$\mathrm{E}$} & FourPeople & -0.04 & -0.05 & -40.1 \\
\hline & Johnny & -0.1 & -0.06 & -38.7 \\
\hline & KristenAndSara & -0.06 & -0.07 & -40.2 \\
\hline \multicolumn{2}{|c|}{ average } & 1.6 & -0.05 & -40.9 \\
\hline
\end{tabular}

\section{CONCLUSIONS}

In this paper, a fast intra prediction algorithm is presented. The proposed method consider the CU and PU to speed up the coding, which employs the texture complexity of CU and exploits the similar three-step search to reduce the intra candidates of PU. Simulation results show that the encoding computational complexity can be reduced by $40.9 \%$ on average and up to $44.1 \%$ compares to the HM software. The proposed method proved it can provide a good performance.

\section{ACKNOWLEDGMENTS}

Our work are sponsored by Program of the National "863" Project (No.2012AA01A309), the integration project of production teaching and research by Guangdong Province and ministry of education (No.2012B09110049 5;No.2009B090300267), the basic research program of Shenzhen City (No.JCYJ20120613113419607), the key project of Shenzhen Polytechnic (No.2213k3190007).

\section{REFERENCES}

[1] B. Bross, W.-J. Han, J.-R. Ohm, G. Sullivan, and T. Wiegand," High efficiency video coding (HEVC) text specification draft 10," Joint Collaborative Team on Video Coding (JCT-VC) of ITU-T SG16 WP3 and ISO/IECJTC1/SC29/WG11,JCTVC-L1003-v29, Geneva,CH (2013).

[2] G. J. Sullivan, J.-R. Ohm, W.-J. Han, and T. Wiegand, "Overview of the High Efficiency Video Coding (HEVC) Standard", IEEE Trans.
Circuits and Systems for Video Technology, 22(12), 1649-1668, (Dec. 2012).

[3] F.Bossen, B. Bross, K.Suhring, and D. Flynn, "HEVC Complexity and Implementation Analysis," IEEE Trans. Circuits and Systems for Video Technology, 22(12) (December 2012).

[4] J.-R. Ohm, G. J. Sullivan, H. Schwarz, T. K. Tan, and T. Wiegand, "Comparison of the Coding Efficiency of Video Coding Standards Including High Efficiency Video Coding (HEVC)", IEEE Trans. Circuits and Systems for Video Technology, 22(12), 1669-1684, (Dec. 2012).

[5] Lainema, J. Bossen, F.; Woo-Jin Han; Junghye-Mim, Ugur, K.,'Intra Coding of the HEVC Standard" IEEE Transactions on Circuits and Systems for Video Technology, 22(12), 1792-1801,(Dec.2012).

[6] Y. Piao, J. Min, and J. Chen, "Encoder improvement of unified intra prediction", Joint Collaborative Team on Video Coding (JCT-VC) of ITU-T SG16 WP3 and ISO/IEC JTC1/SC29/WG11, JCTVC -C207, Guangzhou, CN(Oct. 2010).

[7] Tan, Yih Han, Yeo, Chuohao; Tan, Hui Li; Li, Zhengguo Guo, ”On residual quad-tree coding in HEVC" 2011 IEEE 13th International Workshop on Multimedia Signal Processing (MMSP), Hangzhou, CN(Oct.2011)

[8] Lainema Jani, Ugur Kemal, "Angular Intra Prediction in High Efficiency Video Coding(HEVC)", 2011 IEEE 13th International Workshop on Multimedia Signal Processing (MMSP), Hangzhou, $\mathrm{CN}($ Oct,2011)

[9] Liang Zhao, Li zhang, Siwei Ma,Debin zhao, Wen Gao,"Further Encoder Improve- ment of intra mode decision", Joint Collaborative Team on Video Coding (JCT-VC) of ITU-T SG16 WP3 and ISO/IEC JTC1/SC29/WG11,JCTVC-D283,Daegu,KR(Jan.2011).

[10] Silva, Thaisa Leal da, Agostini, Luciano Volcan; Cruz, Luis A da Silva ," Fast HEVC intra prediction mode decision based on EDGE direction information", 2012 Proceedings of the 20th European Signal Processing Conference (EUSIPCO),1214-1218(Aug.2012).

[11] VanWallendael,Glenn, VanLeuven,Sebastiaan; "Improved intra mode signaling for HEVC", 2011 IEEE International Conference on Multimedia and Expo (ICME), (July,2011)

[12] Guifen Tian, Satoshi Goto, "Content adaptive prediction unit size decision algorithm for HEVC intra coding", Picture Coding Symposium (PCS) ,405-408(May,2012).

[13] Hao Zhang, Zhan Ma; Fast Intra Prediction for High Efficiency Video Coding, Advances in Multimedia Information Processing - PCM 2012,pp568-577.

[14] Li, Renxiang,Zeng, Bing; Liou, Ming-L Lei, "A new three-step search algorithm for block motion estimation", IEEE Transactions on Circuits and Systems for Video Technology,4(4),438-442,(Aug. 1994).

[15] Joint Collaborative Team on Video Coding (JCT-VC) of ITU-T SG16 WP3 and ISO/IECJTC1/SC29/ WG11,"High Efficiency Video Coding Test Model 8.0",(2012) http://hevc.hhi.fraunhofer.desvn/svn_HEVC Software/branches/HM-8.0-dev/

[16] Frank Bossen. "Common test conditions and software reference configurations", Joint Collaborative Team on Video Coding (JCT-VC) of ITU-T SG16 WP3 and ISO/IECJTC1/SC29/ WG11,JCTVC -E700,Geneva, Switzerland(2011).

[17] Kenta Senzaki et al. "BD-PSNR/Rate computation tool for five data points" Joint Collaborative Team on Video Coding (JCT-VC) of ITU-T SG16 WP3 and ISO/IECJTC1/SC29/WG11,JCTVCB055, Geneva, Switzerland(2010). 